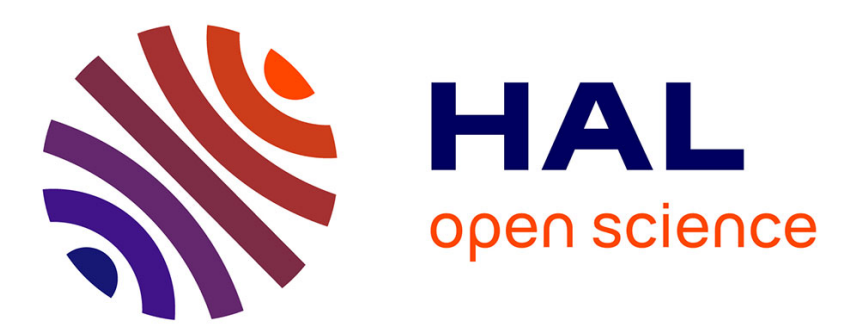

\title{
The excitation of solar-like oscillations in a $\delta$ Sct star by efficient envelope convection
}

\author{
V. Antoci, G. Handler, T. L. Campante, A. O. Thygesen, A. Moya, T.
}

Kallinger, D. Stello, A. Grigahcène, H. Kjeldsen, T. R. Bedding, et al.

\section{- To cite this version:}

V. Antoci, G. Handler, T. L. Campante, A. O. Thygesen, A. Moya, et al.. The excitation of solar-like oscillations in a $\delta$ Sct star by efficient envelope convection. Nature, 2011, 477 (7366), pp.570-573. 10.1038 /nature10389 . cea-00881660

\section{HAL Id: cea-00881660 https://hal-cea.archives-ouvertes.fr/cea-00881660}

Submitted on 24 Sep 2019

HAL is a multi-disciplinary open access archive for the deposit and dissemination of scientific research documents, whether they are published or not. The documents may come from teaching and research institutions in France or abroad, or from public or private research centers.
L'archive ouverte pluridisciplinaire HAL, est destinée au dépôt et à la diffusion de documents scientifiques de niveau recherche, publiés ou non, émanant des établissements d'enseignement et de recherche français ou étrangers, des laboratoires publics ou privés. 


\title{
The excitation of solar-like oscillations in a $\delta$ Sct star by efficient envelope convection
}

\author{
V. Antoci ${ }^{1}$, G. Handler ${ }^{1,2}$, T. L. Campante ${ }^{3,4}$, A. O. Thygesen ${ }^{4,5}$, A. Moya ${ }^{6}$, T. Kallinger ${ }^{1,7,8}$, D. Stello ${ }^{9}$, A. Grigahcène ${ }^{3}$, H. Kjeldsen ${ }^{4}$, \\ T. R. Bedding 9 , T. Lüftinger ${ }^{1}$, J. Christensen-Dalsgaard ${ }^{4}$, G. Catanzaro ${ }^{10}$, A. Frasca ${ }^{10}$, P. De Cat ${ }^{11}$, K. Uytterhoeven ${ }^{12,13,14,15}$, \\ H. Bruntt ${ }^{4}$, G. Houdek ${ }^{1}$, D. W. Kurtz ${ }^{16}$, P. Lenz ${ }^{2}$, A. Kaiser ${ }^{1}$, J. Van Cleve ${ }^{17}$, C. Allen ${ }^{18}$ \& B. D. Clarke ${ }^{17}$
}

Delta Scuti $(\delta \mathrm{Sct})^{1}$ stars are opacity-driven pulsators with masses of 1.5-2.5 $M_{\odot}$, their pulsations resulting from the varying ionization of helium. In less massive stars ${ }^{2}$ such as the Sun, convection transports mass and energy through the outer 30 per cent of the star and excites a rich spectrum of resonant acoustic modes. Based on the solar example, with no firm theoretical basis, models predict that the convective envelope in $\delta$ Sct stars extends only about 1 per cent of the radius ${ }^{3}$, but with sufficient energy to excite solar-like oscillations $^{4,5}$. This was not observed before the Kepler mission ${ }^{6}$, so the presence of a convective envelope in the models has been questioned. Here we report the detection of solar-like oscillations in the $\delta$ Sct star HD 187547, implying that surface convection operates efficiently in stars about twice as massive as the Sun, as the ad hoc models predicted.

Thirty days of continuous observations of HD 187547 (KIC 7548479) by the Kepler mission with a cadence of $1 \mathrm{~min}$ led to its identification as a $\delta$ Sct pulsator (Fig. 1a, b). In contrast to the non-uniformly distributed signals at low frequencies, the observed regularly spaced peaks at high frequencies (Fig. 1c) suggest that we also observe high-radial-order overtones as expected for stochastically excited solar-like oscillations. For such oscillations the observed comb-like frequency structure (with the large frequency separation $\Delta v$ indicating the frequency separation between consecutive radial overtones of like degree) is the result of mainly radial and dipolar pulsation modes, whereas for $\delta$ Sct stars it is not clear which modes are excited to observable amplitudes. The strikingly broadened structures observed only at high frequencies (Figs If and 2b, c) suggest that each is due either to single damped and stochastically re-excited oscillations or to very close unresolved frequencies of coherent oscillations.

Here we use spectroscopic observations to derive an effective temperature $T_{\text {eff }}=7,500 \pm 250 \mathrm{~K}$, a surface gravity of $\log g=3.90 \pm 0.25$ dex (c.g.s.) and a projected rotational velocity of $v \sin i=10.3 \pm 2.3$ $\mathrm{km} \mathrm{s}^{-1}$ (see Supplementary Information for details). We identify HD 187547 as an Am star from chemical element abundance analysis, which is consistent with the observed low $v \sin i$ typical for these stars. Am stars are stars of spectral type A showing atmospheric underabundance when compared with the Sun in the chemical elements $\mathrm{Sc}$ and $\mathrm{Ca}$, and an overabundance of $\mathrm{Ba}, \mathrm{Sr}$ and $\mathrm{Y}$ (ref. 7). We compute a photospheric metallicity (all elements except $\mathrm{H}$ and $\mathrm{He}$ ) of $Z=0.017$, which is larger than the solar value of $Z=0.0134$ (ref. 8).

About two-thirds of Am stars are primary components of spectroscopic binary systems ${ }^{9}$. The Am phenomenon is connected to slow rotation, which is not common in A type stars. Binarity is believed to act as a braking mechanism slowing down the rotation and allowing spectral peculiarities to occur as a result of element diffusion ${ }^{10}$. Pulsating Am stars still represent a challenge to theory, because $\mathrm{He}$ is expected to settle gravitationally and should only partly be present in the He II ionization zone where the $\delta$ Sct pulsations are excited. In other words, theoretical models predict that the hottest and youngest A-type stars should not pulsate ${ }^{10}$, which is in contradiction with recent observations ${ }^{11}$. As the stars evolve, their convective envelopes deepen and efficiently mix the stellar matter, erasing the observed chemical peculiarities in the atmospheres, allowing the opacity mechanism to drive pulsation in the He II ionization zone. Using the observed solarlike oscillations reported here, the depth of the convective envelope can be derived (hence the mixing length), probing the diffusion of $\mathrm{He}$ and heavy elements in this star. This will contribute significantly to revising the interaction between pulsation and diffusion in models of Am stars.

Seven radial velocity measurements of HD 187547, spread over 153 days, give no evidence for a short-period binary system. In addition, the absence of any detectable contribution by a potential close companion to the spectrum implies a considerably less luminous star of spectral type $\mathrm{G}$ or later. The expected amplitudes and frequency of maximum oscillation power for such a star are inconsistent with the observations, leading to the conclusion that the signal observed in Fig. 1c cannot originate from a companion. The observed amplitude spectrum of HD 187547 is not affected by a background star because the fraction of light in the aperture from neighbouring stars is only $1.5 \%$. Other chemically peculiar pulsating stars situated, as the $\delta$ Sct stars, in the classical instability strip in the Hertzsprung-Russell diagram $^{12}$ are the rapidly oscillating Ap stars. Their high-radial-order pulsation modes are triggered by the opacity mechanism acting in the hydrogen ionization zone, often showing equidistant multiplets in the frequency spectrum as a result of the alignment of the pulsation axes with strong magnetic fields ${ }^{13}$. The strong magnetic fields as seen in rapidly oscillating Ap stars are, however, not observed in Am stars ${ }^{14}$. We therefore exclude the possibility that HD 187547 is a hybrid of a $\delta$ Sct and a rapidly oscillating Ap star.

In Fig. 3 we show an échelle diagram comparing the observed frequencies with a model of a star similar to HD 187547, demonstrating again the clear structures separated by $\Delta v$ at high frequencies and the non-structured distribution at lower frequencies. For the high-frequency modes we derive a mean large frequency separation $\Delta v$ of $40.5 \pm 0.6 \mu \mathrm{Hz}$. Using the empirical relation ${ }^{15}$

${ }^{1}$ Institute of Astronomy, University of Vienna, Türkenschanzstraße 18, A-1180 Vienna, Austria. ${ }^{2}$ Copernicus Astronomical Center, Bartycka 18, 00-716 Warsaw, Poland. ${ }^{3}$ Centro de Astrofísica,

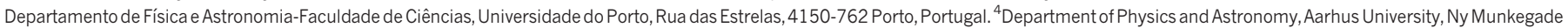

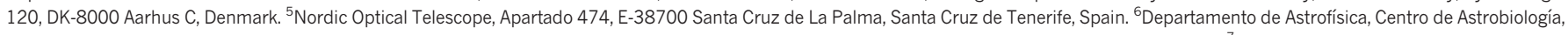
Instituto Nacional de Técnica Aeroespacial - Consejo Superior de Investigaciones Científicas, PO Box 78, E-28691, Villanueva de la Cañada, Madrid, Spain. ${ }^{7}$ Department of Physics and Astronomy,

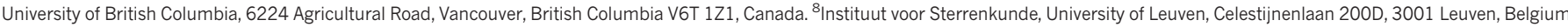

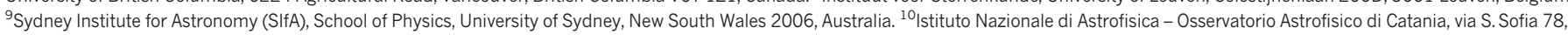

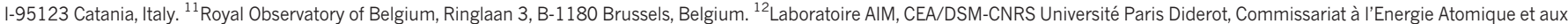
Energies Alternatives, Institut de Recherche sur Les Lois Fondamentales, Service d'Astrophysique, Centre de Saclay, F-91191, Gif-sur-Yvette, France. ${ }^{13}$ Kiepenheuer-Institut für Sonnenphysik, Schöneckstrasse 6, 79104 Freiburg, Germany. ${ }^{14}$ Instituto de Astrofísica de Canarias, 38200 La Laguna, Tenerife, Spain. ${ }^{15}$ Departamento de Astrofísica, Universidad de La Laguna, 38205 La Laguna,

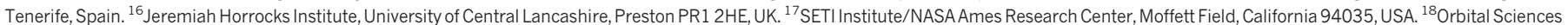
Corporation/NASA Ames Research Center, Moffett Field, California 94035, USA. 

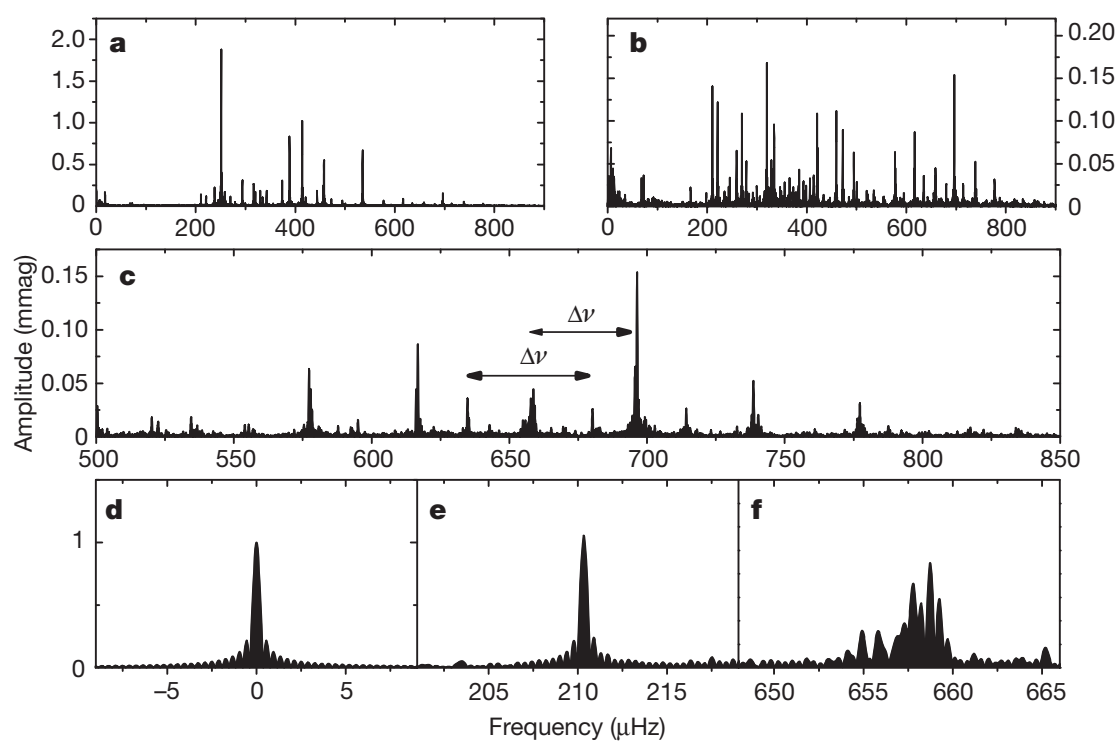

Figure $1 \mid$ Fourier amplitude spectra of the Kepler light curve of

HD 187547. a, Fourier spectrum covering the entire frequency range in which significant signals were observed with a dominant frequency at $251 \mu \mathrm{Hz}$ and an amplitude of 2 mmag, typical for a $\delta$ Sct star. $\mathbf{b}$, The multimode oscillations of HD 187547 are shown by subtracting 16 sinusoids corresponding to the most prominent oscillations, revealing a large number of additional significant frequencies. c, The region between 500 and $850 \mu \mathrm{Hz}$ shows a clear pattern of roughly equally spaced peaks, which we interpret as high-order consecutive radial overtones. The comb-like structure expected for high-order radial

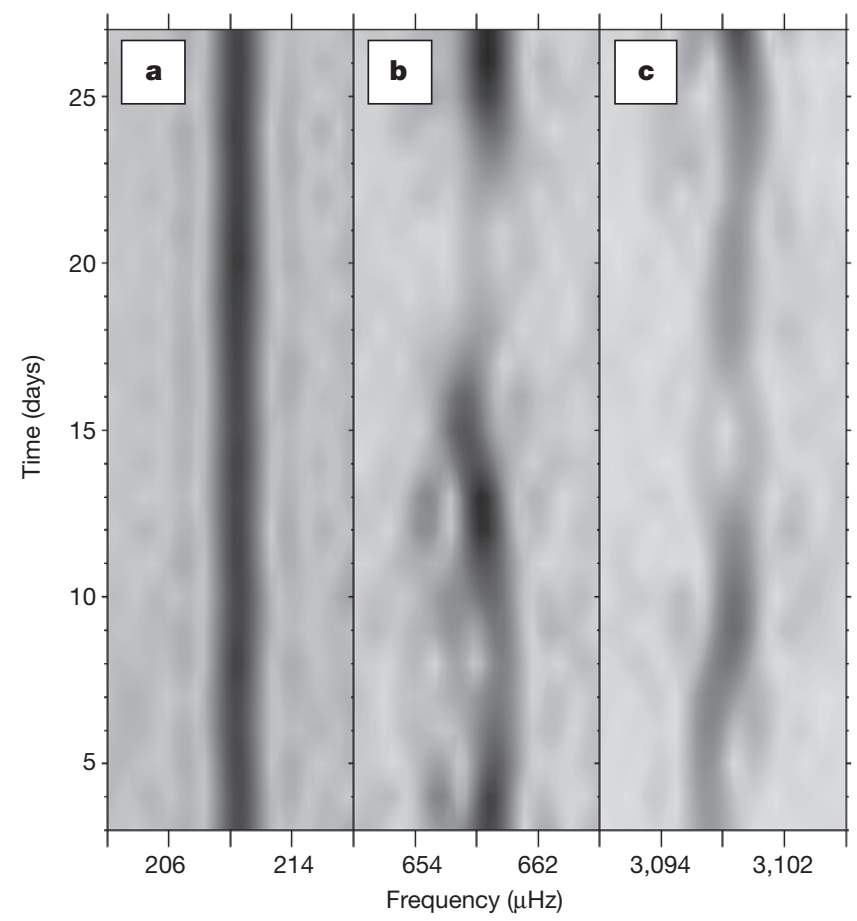

Figure $2 \mid$ Time-Fourier spectrum. Here we again highlight the difference in temporal variability between the modes interpreted as stochastic modes and the coherent, opacity-driven peaks at low frequencies. The time-fourier spectrum was computed with a running filter of full-width at half-maximum $=5$ days, comparable to the mean mode lifetime. a, An opacity-driven mode (the same as in Fig. 1e) showing temporal stability in the $\delta$ Sct frequency region. b. Stochastic mode observed in HD 187547, showing an erratic behaviour as expected for solar-like oscillations (the same as in Fig. 1f). c, For comparison, a stochastic oscillation mode observed in the Sun. The solar data were obtained from the SOHO VIRGO instrument. The data set has the same length and sampling as for HD 187547; that is, 30 days and $1 \mathrm{~min}$, respectively. Further details of frequency analyses and tests on artificial data sets (Supplementary Fig. 1) to verify our interpretation are in the Supplementary Information. overtones is clearly visible. The broadened peaks suggest damped/re-excited solar-like oscillations. The black arrows denoted $\Delta v$ indicate the large separation between consecutive radial and dipole modes. d, Spectral window. The shape of the window function is defined by the length and sampling of the data set. Any coherent signal will have the same profile. e, Example for one of the modes driven by the opacity mechanism in HD 187547.f, A supposed solarlike oscillation mode observed in HD 187547, displaying a broadened structure suggestive of a short mode lifetime.

$\Delta v=(0.263 \pm 0.009) \mu \mathrm{Hz}\left(v_{\max } \mu \mathrm{Hz}^{-1}\right)^{0.772 \pm 0.005}$ we obtain a frequency of maximum power $v_{\max }=682_{-43}^{+41} \mu \mathrm{Hz}$. This is in very good agreement with the highest-amplitude mode in the supposed stochastic frequency region at $696 \mu \mathrm{Hz}$. The possibility that what we observe is $0.5 \Delta v$ in the frequency spectrum is ruled out because this would require a $v_{\max }$ at about $1,673 \mu \mathrm{Hz}$, where no signal is observed. We can also exclude the observation of $2 \Delta v$ because that would place $v_{\max }$ at $277 \mu \mathrm{Hz}$, close to the dominant opacity-driven mode at $251 \mu \mathrm{Hz}$.

The amplitudes of solar-like oscillations are determined by the interaction between driving and damping defined by different physical processes $^{2}$, such as modulation of the turbulent momentum and heat fluxes by pulsation. The exact contribution to driving and damping by each of these processes is still not well understood, resulting in uncertainties in the predictions of the stochastically excited mode amplitudes ${ }^{16}$, particularly in hotter stars ${ }^{2,3}$ in which the convective envelopes are shallow. We expect the mixing length, the amplitudes and mode lifetimes to constrain the anisotropy of the convective velocity field, parameters that all semi-analytical convection models rely on ${ }^{17}$.

For HD 187547 we measure a peak-amplitude per radial mode ${ }^{18}$ for the assumed stochastic signal of $56 \pm 2$ p.p.m., which after bolometric correction $^{19}$ results in $67 \pm 3$ p.p.m. (see Supplementary Information for details). From the empirical scaling relation ${ }^{20}$ and using a bolometric solar peak-amplitude of 3.6 p.p.m. (ref. 21) we obtain a predicted peak amplitude of $A=14 \pm 9$ p.p.m. The mean mode lifetimes are measured $^{22}$ as $5.7 \pm 0.8$ days. Empirical relations predict a mode lifetime for a star with $T_{\text {eff }}=7,500 \pm 250 \mathrm{~K}$ of the order of one day ${ }^{23}$ or shorter ${ }^{24}$, which is not in agreement with what we measure for HD 187547. However, these scaling relations (for amplitude and mode lifetimes) are based on few observed stars, and none of them is calibrated in the temperature domain of our target, for which the physical conditions in the convection zone are expected to be very different. Furthermore, given that HD 187547 is metal overabundant in comparison with the Sun, the observed amplitude is expected to be higher ${ }^{3,25}$ than predicted from simple scaling, which is indeed the case. The power of a mode is directly proportional to the mode lifetime provided that the energy supply rate over the mode inertia is constant ${ }^{26}$, which further supports 


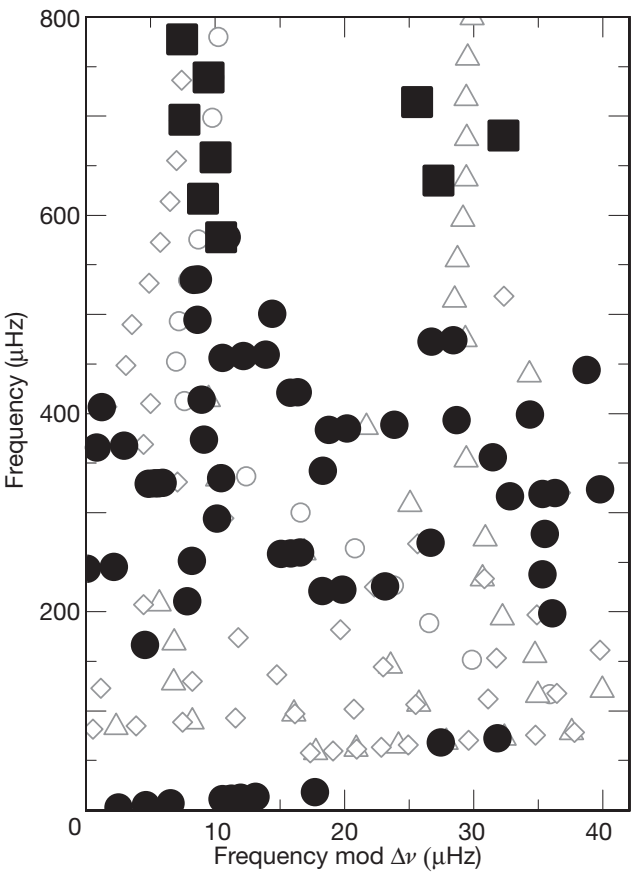

Figure 3 Échelle diagram of HD 187547. Here we plot 69 extracted frequencies as a function of frequency modulo the large separation $(\Delta v=40.5 \mu \mathrm{Hz})$. Frequencies equally spaced by $\Delta v$ will form vertical ridges in the échelle diagram. To guide the eye, we show theoretically predicted frequencies of pulsation modes for a $1.85 M_{\odot}$ stellar model. Ridges of $l=0$ modes are represented by open circles, $l=1$ by open triangles and $l=2$ by open diamonds. Detailed modelling of the star is beyond the scope of this paper. The supposed solar-like modes (filled squares) between 500 and $870 \mu \mathrm{Hz}$ show clear ridges, as expected for high-order acoustic oscillations similar to what is observed in solar-like stars. The lower frequencies that we attribute to $\delta$ Sct pulsation (filled circles) excited by the opacity mechanism show no obvious regular patterns.

the higher amplitude because the observed mode lifetimes are also longer than expected. An additional factor that is not considered in any scaling relations is the chemical peculiarity of our target. In summary, these factors make HD 187547 an intriguing case for further theoretical analyses of stochastic oscillations and the potential interaction with the opacity mechanism in $\delta$ Sct stars.

The amplitude distribution for stochastic pulsation can be described as a Rayleigh distribution, provided that the examined time series are much shorter than the mode lifetimes. The relation between the mean amplitude $\langle A\rangle$ and its standard deviation $\sigma(A)$ can then be written as ${ }^{27}$ $(4 / \pi-1)^{0.5}\langle A\rangle \approx 0.52\langle A\rangle$. This is not valid for opacity-driven pulsation. For HD 187547 we therefore expect to obtain two different regimes of the ratio $\sigma(A) /\langle A\rangle$ for the two groups of oscillation modes (Supplementary Fig. 2). Indeed, we see that the $\delta$ Sct frequencies have a lower value of $\sigma(A) /\langle A\rangle$ than the supposed solar-like modes, giving further evidence for the stochastic nature of the latter (see Supplementary Information for details).

We cannot strictly exclude the possibility that the signals between 578 and $868 \mu \mathrm{Hz}$ are due to unresolved modes of pulsation excited by the opacity mechanism, because high-radial-order acoustic modes can also be observed in hot $\delta$ Sct stars. Nevertheless, as shown in Fig. 1 this would imply that $\delta$ Sct pulsation covers the region between 205 and $870 \mu \mathrm{Hz}$ continuously. According to current theory, the opacity mechanism acting in the He II ionization zone cannot excite modes spanning 16 radial orders for a star with parameters like those of HD 187547 (ref. 28). Further support for the discovery of solar-like oscillations comes from spectroscopic observations that also indicate the presence of convective motions in the atmospheres of $\mathrm{A}$ and $\mathrm{Am} \mathrm{stars}{ }^{29}$. In addition, signatures of granulation noise in $\delta$ Sct stars have been reported from photometric measurements ${ }^{3}$. Opacity-driven pulsations are also observed in more massive stars $\left(8-16 M_{\odot}\right)$, known as $\beta$ Cephei stars (in this case the opacity mechanism acts in the ionization region of the iron-group elements). The unexpected detection of solar-like oscillations in such a $\operatorname{star}^{30}$ (with a mass of $10 M_{\odot}$ ) suggests that both types of pulsation, opacity-driven and stochastically excited, can coexist and can have overlapping frequency domains. The similar timescales of the different oscillation types imply a possible interaction between the two mechanisms.

Received 7 April; accepted 26 July 2011.

Published online 14 September; corrected 29 September 2011 (see full-text HTML version for details).

1. Breger, M. in Delta Scuti and Related Stars (ASP Conf. Ser. Vol. 210) (eds Breger, M. \& Montgomery, M.) 3-42 (Astronomical Society of the Pacific, 2000).

2. Chaplin, W. J. et al. Ensemble asteroseismology of solar-type stars with the NASA Kepler mission. Science 332, 213-216 (2011).

3. Kallinger, T. \& Matthews, J. M. Evidence for granulation in early A-type stars. Astrophys. J. 711, L35-L39 (2010).

4. Houdek, G., Balmforth, N. J., Christensen-Dalsgaard, J. \& Gough, D. O. Amplitudes of stochastically excited oscillations in main-sequence stars. Astron. Astrophys. 351, 582-596 (1999).

5. Samadi, R., Goupil, M.-J. \& Houdek, G. Solar-like oscillations in delta Scuti stars. Astron. Astrophys. 395, 563-571 (2002).

6. Koch, D. G. et al. Kepler mission design, realized photometric performance, and early science. Astrophys. J. 713, L79-L86 (2010).

7. Preston, G. W. The chemically peculiar stars of the upper main sequence. Annu. Rev. Astron. Astrophys. 12, 257-277 (1974).

8. Asplund, M., Grevesse, N., Sauval, A. J. \& Scott, P. The chemical composition of the Sun. Annu. Rev. Astron. Astrophys. 47, 481-522 (2009).

9. Carquillat, J.-M. \& Prieur, J.-L. Contribution to the search for binaries among Am stars. VIII. New spectroscopic orbits of eight systems and statistical study of a sample of 91 Am stars. Mon. Not. R. Astron. Soc. 380, 1064-1078 (2007).

10. Turcotte, S., Richer, J., Michaud, G. \& Christensen-Dalsgaard, J. The effect of diffusion on pulsations of stars on the upper main sequence $-\delta$ Scuti and metallic A stars. Astron. Astrophys. 360, 603-616 (2000).

11. Balona, L. et al. Kepler observations of Am stars. Mon. Not. R. Astron. Soc. 414, 792-800 (2011).

12. Handler, G. Confirmation of simultaneous p and g mode excitation in HD 8801 and $\gamma$ Peg from time-resolved multicolour photometry of six candidate 'hybrid' pulsators. Mon. Not. R. Astron. Soc. 398, 1339-1351 (2009).

13. Kurtz, D. W. Rapidly oscillating AP stars. Mon. Not. R. Astron. Soc. 200, 807-859 (1982).

14. Aurière, M. et al. No detection of large-scale magnetic fields at the surfaces of Am and HgMn stars. Astron. Astrophys. 523, A40, doi:10.1051/0004-6361/ $201014848(2010)$

15. Stello, D., Chaplin, W. J., Basu, S., Elsworth, Y. \& Bedding, T. R. The relation between $\Delta v$ and $v_{\max }$ for solar-like oscillations. Mon. Not. R. Astron. Soc. 400L, L80-L84 (2009).

16. Houdek, G. Solar-type variables. AIP Conf. Proc. 1170, 519-530 (2009).

17. Samadi, R., Belkacem, K., Goupil, M.-J., Kupka, F. \& Dupret, M.-A. Solarlike oscillation amplitudes and line-widths as a probe for turbulent convection in stars. Proc. Int. Astron. Un. 239, 349-357 (2007).

18. Kjeldsen, H. et al. The amplitude of solar oscillations using stellar techniques. Astrophys. J. 682, 1370-1375 (2008).

19. Ballot, J., Barban, C. \& van't Veer-Menneret, C. Visibilities and bolometric corrections for stellar oscillation modes observed by Kepler. Astron. Astrophys. 531, A124, doi:10.1051/0004-6361/201016230 (2011).

20. Kjeldsen, H. \& Bedding, T. R. Amplitudes of solar-like oscillations: a new scaling relation. Astron. Astrophys. 529, L8, doi:10.1051/0004-6361/201116789 (2011).

21. Michel, E. et al. Intrinsic photometric characterisation of stellar oscillations and granulation. Astron. Astrophys. 495, 979-987 (2009).

22. Gruberbauer, M., Kallinger, T., Weiss, W. W. \& Guenther, D. B. On the detection of Lorentzian profiles in a power spectrum: a Bayesian approach using ignorance priors. Astron. Astrophys. 506, 1043-1053 (2009).

23. Chaplin, W. J., Houdek, G., Karoff, C., Elsworth, Y. \& New, R. Mode lifetimes of stellar oscillations. Implications for asteroseismology. Astron. Astrophys. 500, L21-L24 (2009).

24. Baudin, F. et al. Amplitudes and lifetimes of solar-like oscillations observed by CoRoT. Red-giant versus main-sequence stars. Astron. Astrophys. 529, A84, doi:10.1051/0004-6361/201014037 (2011).

25. Samadi, R., Ludwig, H.-G., Belkacem, K., Goupil, M. J. \& Dupret, M.-A. The CoRoT target HD 49933. I. Effect of the metal abundance on the mode excitation rates. Astron. Astrophys. 509, A15, doi:10.1051/0004-6361/200911867 (2010).

26. Chaplin, W. J. et al. On model predictions of the power spectral density of radial solar p modes. Mon. Not. R. Astron. Soc. 360, 859-868 (2005).

27. Chang, H.-Y. \& Gough, D. O. On the power distribution of solar p modes. Solar Phys. $181,251-263(1998)$

28. Pamyatnykh, A. A. in Delta Scuti and Related Stars (ASP Conf. Ser. Vol. 210) (eds Breger, M. \& Montgomery, M.) 215-246 (Astronomical Society of the Pacific, 2000). 
29. Landstreet, J. D. et al. Atmospheric velocity fields in tepid main sequence stars Astron. Astrophys. 503, 973-984 (2009).

30. Belkacem, K. et al. Solar-like oscillations in a massive star. Science $\mathbf{3 2 4}$, 1540-1542 (2009).

Supplementary Information is linked to the online version of the paper at www.nature.com/nature.

Acknowledgements We thank the entire Kepler team, without whom these results would not be possible. V.A., G.Ha. and G.Ho. were supported by the Austrian Fonds zur Förderung der wissenschaftlichen Forschung. V.A. also thanks L. Fossati for his help. A.M. acknowledges the funding of AstroMadrid, who was also supported by Spanish grants. T.R.B. and D.S. acknowledge support from the Australian Reseach Council. T.L was supported by the Austrian Agency for International Cooperation in Education and Research. K.U. acknowledges financial support from the Deutsche

Forschungsgemeinschaft. Funding for this Discovery mission is provided by NASA's Science Mission Directorate.

Author Contributions V.A. discovered the star among the Kepler targets, analysed it and found the solar-like oscillations (as a part of her PhD thesis), did spectroscopic analyses, frequency analyses, the test on the stochastic nature of the signal, and interpretations, and wrote the paper. G.Ha. had the idea for this project and supervised V.A., helped with analyses, interpretations and writing the paper.T.L.C. contributed to the analyses of the stochastic modes and also to the test on the stochastic nature of the signal. A.O.T. observed the target spectroscopically at Nordic Optical Telescope, identified the star as an Am star and did spectroscopic analyses. A.M. contributed to the statistical test on the nature of the stochastic signal. T.K. helped interpretations, data analyses and writing the paper, and delivered the mode lifetimes. D.S. helped with data analyses, writing the paper and made Fig. 2. A.G. helped with theoretical interpretations and the time-Fourier analyses. T.R.B. helped with interpretations and writing the paper. H.K. contributed to the analyses, also by supervising V.A., and is a member of the Kepler Asteroseismic Investigation Steering Committee. J.C-D. helped with the theoretical support, writing the paper and is a member of the Kepler Asteroseismic Investigation Steering Committee. T.L. confirmed the Am identification, excluding the Ap character of the star. G.C., A.F. and A.K. did spectroscopic analyses. P.D.C. was Principal Investigator and observer for the spectroscopic data from McDonald observatory. K.U. was Co-Investigator of the McDonald data and coordinated the ground-based observations. H.B. was Principal Investigator for the observations with the NARVAL spectrograph and did spectroscopic analyses. G.Ho. and P.L. helped with theoretical interpretations and writing the paper. D.W.K. helped with the Am classification and writing the paper and is leader of the delta Scuti working group of the Kepler Asteroseismic Science Consortium. J.V.C., C.A. and B.D.C. are part of the Kepler team and were involved in designing and operating the satellite. All co-authors contributed to discussions and commented on the manuscript.

Author Information Reprints and permissions information is available at www.nature.com/reprints. The authors declare no competing financial interests. Readers are welcome to comment on the online version of this article at www.nature.com/nature. Correspondence and requests for materials should be addressed to V.A. (victoria.antoci@univie.ac.at). 